Check for updates

Cite this: RSC Adv., 2021, 11, 36748

Received 22nd September 2021 Accepted 9th November 2021

DOI: $10.1039 / d 1 r a 07092 d$

rsc.li/rsc-advances

\section{One-pot synthesis of new alkyl 1-naphthoates bearing quinoline, pyranone and cyclohexenone moieties via metal-free sequential addition/ oxidation reactions $\uparrow$}

\author{
Seyedeh Hekmat Mousavi, ${ }^{a}$ Mohammad Reza Mohammadizadeh, ${ }^{a}$

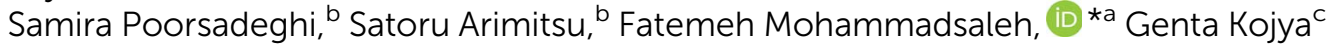 \\ and Shinichi Gima ${ }^{c}$
}

\begin{abstract}
A mild and one-pot synthetic pathway was successfully developed for the synthesis of new naphthoatebased scaffolds containing quinoline, pyranone and cyclohexenone moieties via a multistep reaction between acenaphthoquinone and various 1,3-diketones in the presence of different primary aliphatic and benzylic alcohols. This reaction proceeds via a sequential addition/oxidation mechanistic process including a metal-free addition step of acenaphthoquinone and 1,3-diketones followed by the $\mathrm{H}_{5} \mid \mathrm{O}_{6}{ }^{-}$ mediated $\mathrm{C}-\mathrm{C}$ oxidative cleavage of the corresponding vicinal diols at room temperature. The alcohols play a dual role, as the reaction solvent as well as the nucleophile, to conduct the reaction process toward naphthoate formation. All alkyl naphthoate derivatives prepared in this work are new compounds and were definitively characterized using ${ }^{1} \mathrm{H}-\mathrm{NMR},{ }^{13} \mathrm{C}-\mathrm{NMR}$ and HRMS analysis, while $\mathrm{X}$-ray crystallography was carried out for one of the products. The synthesis of a naphthalene-based nucleus attached to heterocyclic moieties is noteworthy to follow in the near future for diverse applications in biology, medicine, metal complex design, and semiconductor and optical materials.
\end{abstract}

\section{Introduction}

Naphthalene and its derivatives are important organic platforms which are used extensively in industrial chemical compounds and in the chemistry of various pharmacology agents including anticancer, ${ }^{\mathbf{1 , 2}}$ antiviral $^{\mathbf{3}}$ and anti-inflammatory ${ }^{4}$ agents and the production of synthetic plastics, ${ }^{5}$ organic semiconductor materials $^{6}$ and optics. ${ }^{7}$

Heterocyclic compounds are of very much interest to medicinal chemists and many heterocyclic scaffolds have demonstrated unique biological properties. ${ }^{8-10}$ Due to their applications in various fields of chemistry and material sciences, ${ }^{4}$ naphthalene-based heterocyclic compounds have attracted many attentions. Large number of heterocyclic naphthalene-based scaffolds has shown significant and

${ }^{a}$ Department of Chemistry, Faculty of Nano and Bioscience and Technology, Persian Gulf University, Bushehr 75169, Islamic Republic of Iran. E-mail: $f$. mohammadsaleh@gmail.com; Fax: 987731223348

${ }^{b}$ Department of Chemistry, Biology and Marine Science, Faculty of Science, University of the Ryukyus, 1-Senbaru, Nakagami, Nishihara, Okinawa, 903-0213, Japan

${ }^{c}$ Center for Research Advancement and Collaboration, University of the Ryukyus, Senbaru 1, Nishihara, Okinawa 903-0213, Japan

$\dagger$ Electronic supplementary information (ESI) available. CCDC 2110682. For ESI and crystallographic data in CIF or other electronic format see DOI: 10.1039/d1ra07092d satisfactory biological action. ${ }^{\mathbf{1 1 - 1 3}}$ Several naphthalene-based structures have been marketed as medicine and approved by FDA $^{\mathbf{1 4 , 1 5}}$ (Fig. 1). However, much studies need to be conducted to the synthesis of new naphthalene scaffolds and the potential of these compounds should be more discovered through extensive research.

Bhati has recently designed a series of naphthalene based SARS-CoV PLpro inhibitors by linking naphthalene scaffold to the 3,4-dihydro-2H-pyran moiety via-NHCO functional group. ${ }^{\mathbf{1 6}}$ PLpro is an essential enzyme for coronaviruses to express and replicate their genomic and it is very important for targeting

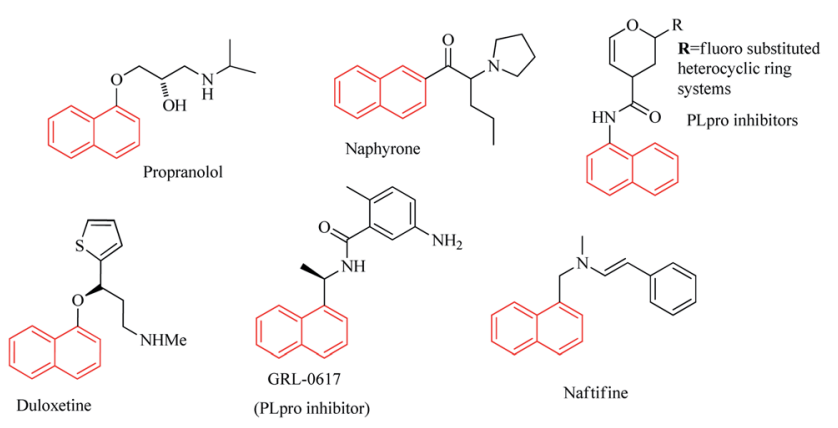

Fig. 1 Structural examples for some naphthalene containing drugs. 
PLpro to treat coronavirus infections. The ongoing global pandemic of coronavirus disease 2019 (COVID-19) has influenced all the sections of the society and as the most immediate problem of the world has created an excessive social economic and health care challenge.

Some recent literatures have reported the naphthalenebased PLpro inhibitors and confirmed their potential as COVID-19 therapeutics. ${ }^{\text {15-20 }}$ Naphthalene based PLpro inhibitors were found to be effective at blocking SARS-CoV-2 (COVID19) PLpro activity as well as SARS-CoV-2 replication. ${ }^{21}$

Ersan reported the synthesis of new naphthalene utilizing heterocyclic moieties and studied their antimicrobial activity. ${ }^{\mathbf{1 1}}$ These compounds showed high potential in designing new inhibitors of $E$. coli topoisomerase I. Naphthoates as important derivatives of naphthalene exist in many natural and synthetic biologically active materials and their derivatives demonstrate various applications including biological activity, ${ }^{22}$ optoelectronic properties, ${ }^{23}$ ligand and metal-chelator. ${ }^{3,24}$ Liou prepared several methyl naphthalene carboxylates (naphthoates) and evaluated their anti-inflammatory activities by superoxide anion generation and elastase release. ${ }^{25}$ New derivatives of naphthoates have been widely used as ligands and efficient chelators for metal-complex formation with a variety of metals including $\mathrm{Cu},{ }^{26,27} \mathrm{Cd},{ }^{28,29} \mathrm{~Pb}^{24}$ and etc. Dai reported the synthesis and X-ray single-crystal structure analysis of new naphthoate-based cadmium(II) and lead(II) complexes. ${ }^{\mathbf{2 4 , 2 9}}$ Ahmad Irfan $^{7}$ synthesized Schiff based naphthalene compounds and studied the effect of electron donor/acceptor groups on the electro-optical, charge transfer and NLO properties of products by DFT and TDDFT. Due to the electron injection, electronic coupling constant and light harvesting efficiency they concluded that the studied Schiff base compounds would be good contestants to be used in dye-sensitized solar cells. Based on these results, the heterocyclic scaffolds containing naphthalene ring as fully $\pi$-conjugated materials are good candidate for optoelectronic applications and organic semiconductor materials. $\mathrm{Chen}^{26}$ prepared the naphthoate-modifying $\mathrm{Cu}^{2+}$-detective Bodipy sensors with the fluorescent $\mathrm{ON}-\mathrm{OFF}$ performance. Their results reveal the effect of the naphthoated esterification on the fluorescence emission of the products.

With these considerations in mind, and in the continuation of our efforts to develop new pathways toward the chemical synthesis ${ }^{30-36}$ and as part of our current study on the oxidative cleavages of cyclic vicinal diols to synthesize new potentially biologically active heterocycles, ${ }^{31}$ we have herein focused on the development of a facile and effective method for the synthesis of new naphthoate derivatives (Scheme 1). In this study, we report the results of our efforts on the synthesis of various new alkyl 1-
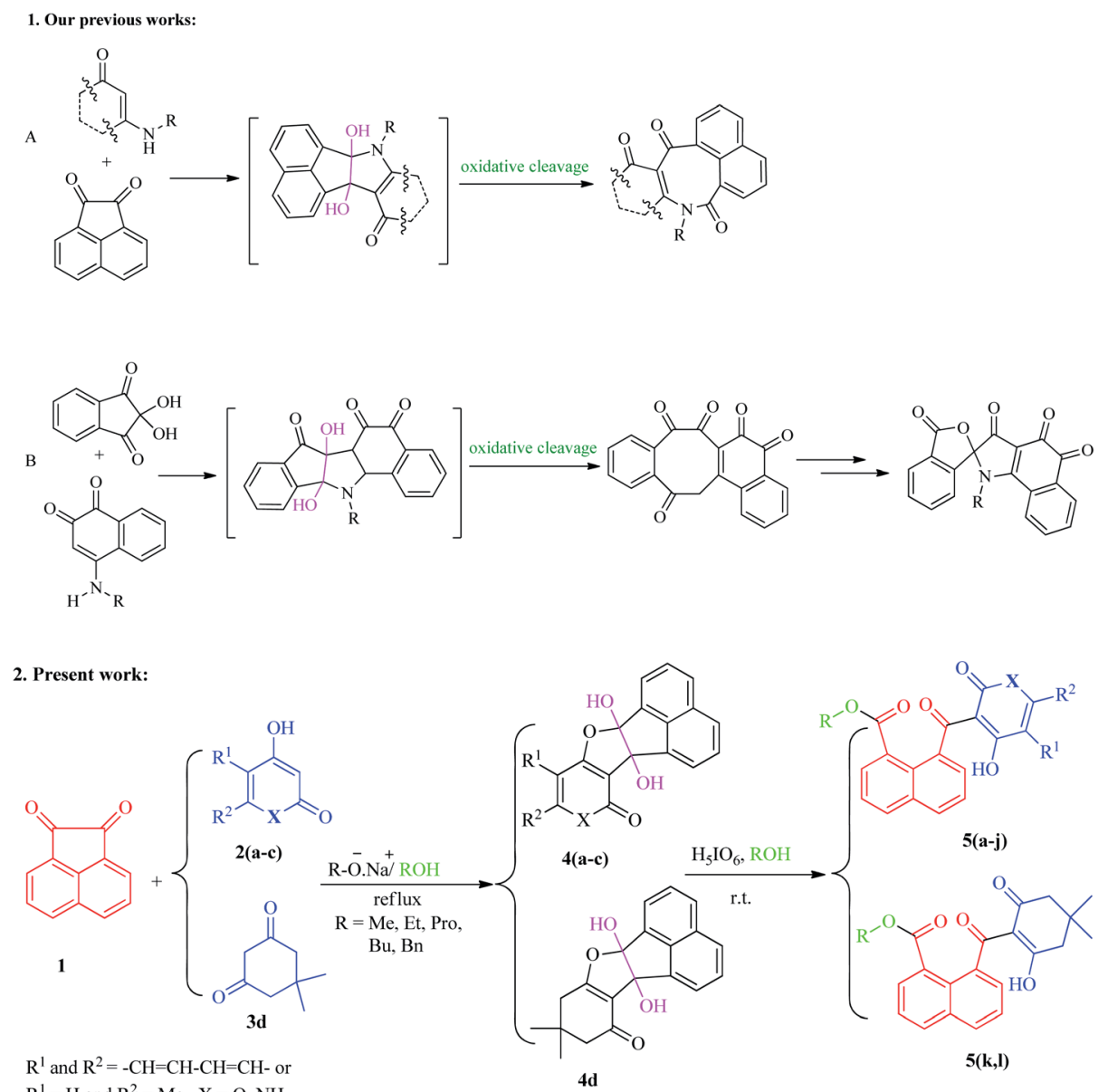

Scheme 1 Our synthetic methods on the preparation of various heterocyclic and non-heterocyclic compounds including the $\mathrm{C}-\mathrm{C}$ oxidative cleavage of cyclic vicinal diols intermediates. 
naphthoates containing 2,4-dihydroxyquinoline, 4-hydroxy-2pyranone, and 2-hydroxycyclohexenones, starting from acenaphthoquinone and various 1,3-diketones in the presence of different primary alcohols $(\mathrm{ROH}, \mathrm{R}$ : aliphatic and benzylic groups) as solvent and reagent followed by metal-free $\mathrm{H}_{5} \mathrm{IO}_{6}$ mediated oxidative cleavage of the corresponding vicinal diols. The different naphthoate derivatives bearing $\mathrm{N}$ - or Oheterocyclic and non-heterocyclic parts were synthesized with good to excellent yields under mild reaction conditions.

\section{Results and discussion}

As shown in the Scheme 1, the various naphthoate derivatives (5a-1) were synthesized in two sequential steps involving addition step resulting to the formation of vicinal cyclic dihydroxy intermediates 4 and $\mathrm{C}-\mathrm{C}$ oxidative cleavage process as a one-pot reaction. In the addition step, acenaphthoquinone $\mathbf{1}$ reacted with various derivatives of $\beta$-diketones 2 and 3 in different aliphatic and benzylic alcohols under reflux conditions and in the presence of sodium alkoxide (conjugate base of each alcohol).

As described in our previous works, vicinal diols are produced as the main intermediates in the addition reactions between different dinucleophiles and diketones. ${ }^{31,37}$ The $\mathrm{C}-\mathrm{C}$ bond oxidative cleavage of vicinal diols has been studied in many literatures. ${ }^{38-40}$ Vicinal diols are cleaved by oxo-donor reagents such as periodic acid to yield carbonyl-containing derivatives via oxo-transfer mechanism, depending on the reaction conditions, reagents, and the number of groups substituted on the carbon atoms bearing the hydroxyl groups. In this study, no attempts were carried out for separation of diols intermediates $\mathbf{4}$, thus, after the addition step, they directly treated with $\mathrm{H}_{5} \mathrm{IO}_{6}$ as the oxidant reagent and the reaction was allowed to continue at room temperature.

Both reaction steps were performed as one-pot in the presence of various alcohols, in which the alcohol plays a dual role as the reaction solvent as well as nucleophiles after the oxidative cleavage stage to produce the final naphthoate derivatives. Both aliphatic and benzylic alcohols were found to be successful in this reaction process and resulted in the formation of different naphthoate products (5a-1) (Scheme 2).

We also studied the scope and limitation of this synthetic methodology using different cyclic $\beta$-dicarbonyls such as dimedone $(\mathbf{5 k}, \mathbf{l}), 4$-hydroxycoumarin (5a-c), 4-hydroxyquinoline (5d-f) and 4-hydroxy-6-methyl-2-pyrone (5g-j). As shown in Scheme 3, the corresponding naphthoate derivatives were

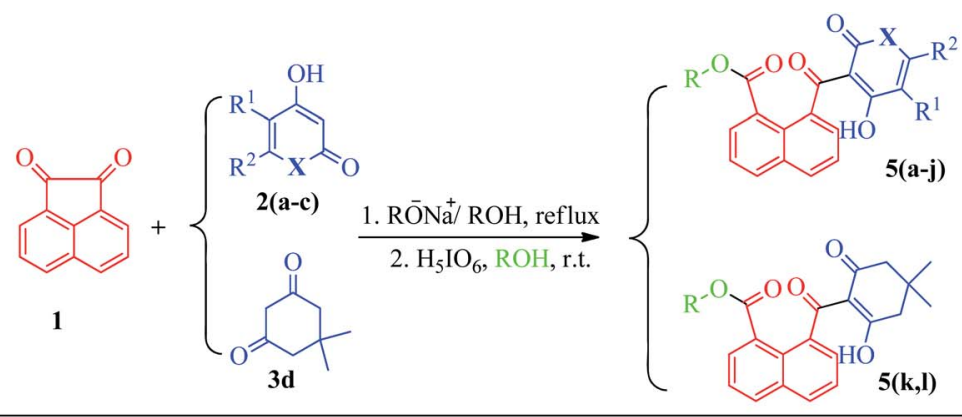

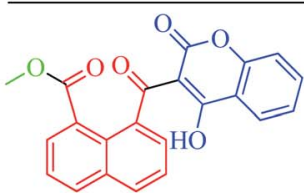

$5 \mathrm{a}(95 \%)$

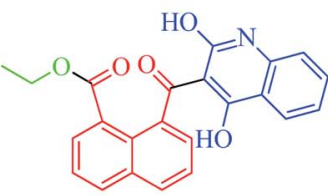

5e $(95 \%)$

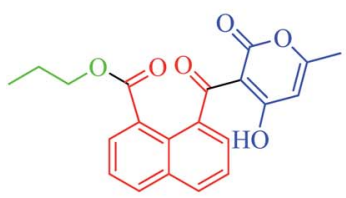

5 i $(95 \%)$

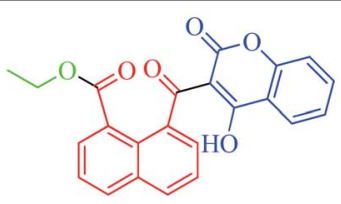

$5 b(92 \%)$

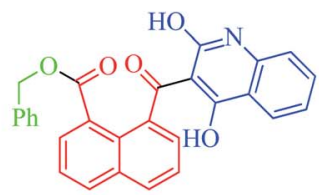

5f $(85 \%)$

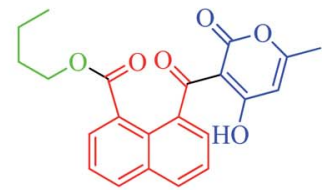

$5 \mathrm{j}(84 \%)$

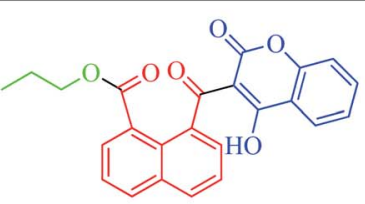

$5 c(88 \%)$

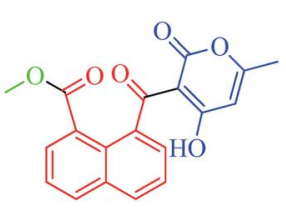

$5 g(90 \%)$

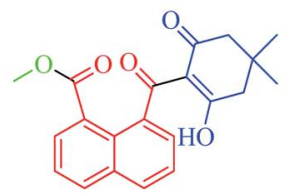

$5 k(80 \%)$

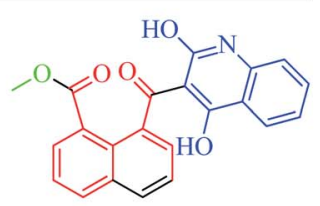

$5 d(90 \%)$

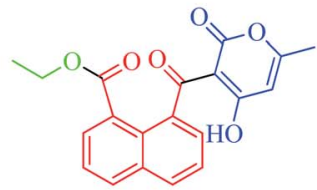

5 h $(96 \%)$

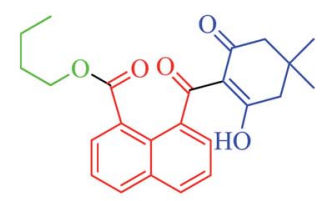

$51(80 \%)$

Scheme 2 Reaction conditions: $\beta$-diketones $(1 \mathrm{mmol})$, acenaphthoquinone $(1 \mathrm{mmol})$; step 1: $\mathrm{Na}(0.3 \mathrm{mmol}), \mathrm{ROH}(3 \mathrm{ml}), \mathrm{reflux}, 12 \mathrm{~h}$; step 2: $\mathrm{H}_{5} \mathrm{IO}_{6}(1.1 \mathrm{mmol})$, r.t., $\mathrm{ROH}, 30-60 \mathrm{~min}$. 
successfully obtained in good to excellent yields, indicating that the type and structure of $\beta$-dicarbonyls had no significant effect on the reaction rates, however, under the same reaction conditions, the use of heterocyclic $\beta$-dicarbonyls 4-hydroxycoumarin, 4-hydroxyquinoline and 4-hydroxy-6-methyl-2pyrone gave higher yields of the corresponding products $(\mathbf{5 a}-\mathbf{j})$ than those for dimedone $(\mathbf{5 k}, \mathbf{l})$. All the final products were purified by recrystallization and fully characterized by recording their spectral data using ${ }^{1} \mathrm{H}-\mathrm{NMR},{ }^{13} \mathrm{C}-\mathrm{NMR}$ as well as highresolution mass (HRMS) analysis.

For a typical example, the ${ }^{1} \mathrm{H}-\mathrm{NMR}$ spectrum of $\mathbf{5 d}$ in $\mathrm{CDCl}_{3}$ displayed three singlets, at 3.64, 11.52, and $15.92 \mathrm{ppm}$, which were attributed to the methoxy and the $\mathrm{OH}$ groups of quinolone ring, respectively. The aromatic protons were also characterized by the presence of two doublets $(\delta=6.48, J=8.4 \mathrm{~Hz} ; \delta=7.68, J$ $=7.2 \mathrm{~Hz})$, two triplets $(\delta=7.19, J=7.6 \mathrm{~Hz} ; \delta=7.41, J=8.0)$, one doublet-doublet $(\delta=7.97, J=7.2 \mathrm{~Hz}, J=1.6 \mathrm{~Hz})$ and two multiplet peaks at $\delta=7.52-7.57$ and $\delta=8.03-8.13 \mathrm{ppm}$. The ${ }^{13} \mathrm{C}-\mathrm{NMR}$ spectrum of $\mathbf{5 d}$ exhibited 22 characteristic signals, whereas methoxy carbon at $\delta=52.9$ and carbonyl carbons were observed at $\delta 169.4$ and $\delta$ 201.8. The aromatic carbons were appeared at the region of 114.6-140.7. The mass analysis of $\mathbf{5 d}$ displayed an $[\mathrm{M}+\mathrm{H}]^{+}$peak at $m / z=374.0964$, which is in agreement with the proposed structure. We also recorded a single crystal X-ray crystallographic analysis of compound $\mathbf{5 d}$ for unambiguous structure determination, which demonstrated the exact structure of this product (Fig. 2).

According to our results and the other reports, ${ }^{31,41}$ we have proposed a plausible mechanism for this synthetic process as depicted in Scheme 3, in which the alkoxide ion acts as the base to activate the 1,3-diketone 2 , which subsequently attacks acenaphthoquinone $\mathbf{1}$ to produce the vicinal diol intermediate $\mathbf{4}$. Next, the vicinal hydroxyl containing intermediate 4 is oxidatively cleaved by periodic acid to form the intermediate II passed from furo[2,3-c][1,2,5]iodadioxole-2,2,2-triol intermediate $\mathbf{I}^{42}$ Due to $\mathrm{sp}^{2}$ hybridization of all carbon atoms, specially, existence of rigid acenaphthylene moiety, the intermediate II suffers from significant strain and prone to ring-opening
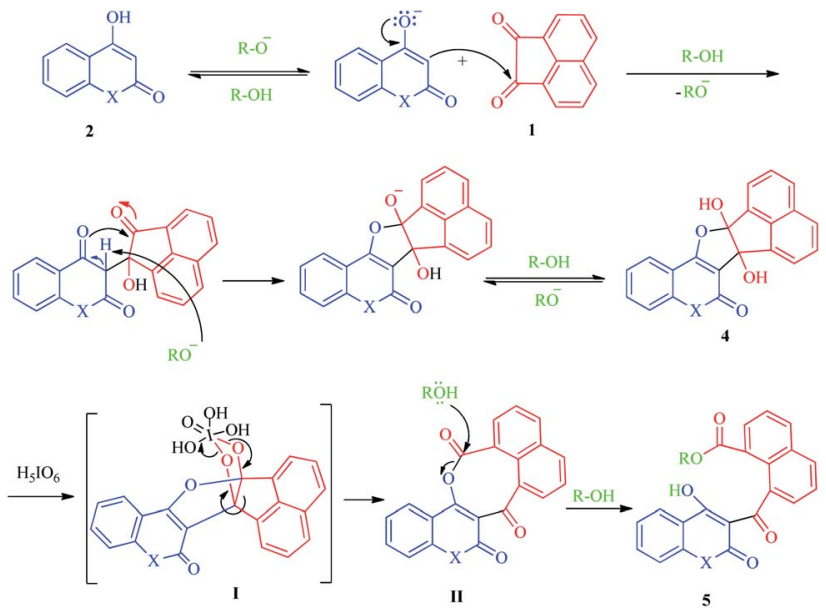

Scheme 3 Proposed mechanism for the synthesis of naphthoate derivatives 5 .

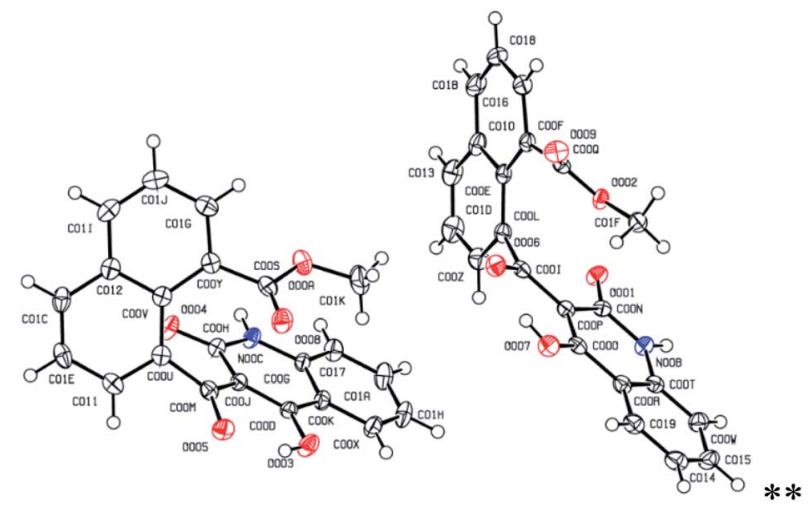

Fig. 2 Single-crystal X-ray representation of $5 d$.

reactions. Thus, it undergoes solvolysis with $\mathrm{ROH}$ to produce the final naphthoate derivatives 5. Due to the growing importance of naphthalene-containing compounds in many areas of chemistry and biology, the development of high-yielding and selective synthetic procedures for the synthesis of these versatile platforms will be significantly considered by researchers.

\section{Conclusions}

A variety of new naphthoate derivatives was synthesized via a mild, eco-friendly, high-yielding and efficient methodology, in which the various 1,3-diketones reacted with acenaphthoquinone by the sequential addition/oxidation mechanistic reaction process in the presence of $\mathrm{RO}^{-} / \mathrm{ROH}$. The reaction processes were conducted as metal-free one-pot reaction in two steps. After the addition stage which performed in alcoholic solvents under reflux, the oxidative cleavage step was carried out in the presence of periodic acid at room temperature. The $\mathrm{ROH}$ acts as a solvent as well as $O$-nucleophiles and conducts the reaction to the formation of the products. The new naphthoates were purified and fully characterized. In general, reaction rate, reaction condition and selectivity of the products are important advantages that must be considered in this transformation.

\section{Experimental}

\section{General information}

The chemicals used in this work were purchased from Merck and Sigma-Aldrich chemical companies and were used without further purification. Melting points were determined using an Electro thermal 9100 apparatus. ${ }^{1} \mathrm{H}-\mathrm{NMR}$ and ${ }^{13} \mathrm{C}-\mathrm{NMR}$ spectra were recorded by using a Bruker DRX-400 AVANCE spectrometer in $\mathrm{CDCl}_{3}$ as solvent. IR spectra were recorded using a Shimadzu IR-470 spectrometer with KBr plates.

\section{General procedure for the synthesis of naphthoate derivatives} $(5 a-1)$

To a mixture of sodium $(0.3 \mathrm{mmol})$ and alcohol $(3 \mathrm{ml})$ in a round-bottom flask, 1,3-diketone $(1 \mathrm{mmol})$ and acenaphthoquinone ( $1 \mathrm{mmol}$ ) were added and the mixture was refluxed with stirring for 12 hours. The reaction process was monitored by performing TLC using $n$-hexane/EtOAc. After completion, the 
reaction mixture was cooled to room temperature and periodic acid ( $1.1 \mathrm{mmol})$ was added to the reaction flask and the mixture was stirred at room temperature for about 30-60 min. The final products 5 were formed as the precipitates in the reaction mixture and separated by filtration and recrystallized from their corresponding alcohols to yield pure naphthoate derivatives $\mathbf{5}$.

\section{Conflicts of interest}

All authors declare that they have no conflict of interest associated with this publication.

\section{Acknowledgements}

The authors gratefully acknowledge the financial support received for this work from the Persian Gulf University of Bushehr (PGU), IR, Iran.

\section{Notes and references}

1 L. Luo, J. J. Jia, Q. Zhong, X. Zhong, S. Zheng, G. Wang and L. He, Eur. J. Med. Chem., 2021, 213, 113039.

2 G. Wang, J. Qiu, X. Xiao, A. Cao and F. Zhou, Bioorg. Chem., 2018, 76, 249-257.

3 R. Perrone, F. Doria, E. Butovskaya, I. Frasson, S. Botti, M. Scalabrin, S. Lago, V. Grande, M. Nadai and M. Freccero, J. Med. Chem., 2015, 58, 9639-9652.

4 M. A. Abozeid, A. A. El-Sawi, M. Abdelmoteleb, H. Awad, M. M. Abdel-Aziz, A.-R. H. Abdel-Rahman and E.-S. I. ElDesoky, RSC Adv., 2020, 10, 42998-43009.

5 E. Hu, S. Shang, Z. Fu, X. Zhao, X. Nan, Y. Du and X. Chen, Chemosphere, 2020, 245, 125602.

6 N. Kobayashi, H. Kuwae, J. Oshima, R. Ishimatsu, S. Tashiro, T. Imato, C. Adachi, S. Shoji and J. Mizuno, J. Lumin., 2018, 200, 19-23.

7 A. Irfan, A. Kalam, A. R. Chaudhry, A. G. Al-Sehemi and S. Muhammad, Optik, 2017, 132, 101-110.

8 M. Kidwai, R. Venktaramanan, R. Mohan and P. Sapra, Curr. Med. Chem., 2002, 9, 1209-1228.

9 A. Mermer, T. Keles and Y. Sirin, Bioorg. Chem., 2021, 105076.

10 S. Rani, K. Raheja, V. Luxami and K. Paul, Bioorg. Chem., 2021, 105017.

11 R. H. Ersan, A. Yuksel, T. Ertan-Bolelli, A. Dogen, S. Burmaoglu and O. Algul, J. Chin. Chem. Soc., 2021, 68, 374-383.

12 H. J. Park, C. W. Song, S. Sarkar, Y. W. Jun, Y. J. Reo, M. Dai and K. H. Ahn, Chem. Commun., 2020, 56, 7025-7028.

13 G. Chinnasamy, K. Subramani and V. Srinivasan, Orient. J. Chem., 2017, 33, 1856-1863.

14 S. Makar, T. Saha and S. K. Singh, Eur. J. Med. Chem., 2019, 161, 252-276.

15 A. K. Ghosh, M. Brindisi, D. Shahabi, M. E. Chapman and A. D. Mesecar, ChemMedChem, 2020, 15, 1-27.

16 S. Bhati, Heliyon, 2020, 6, e05558.

17 E. Pitsillou, J. Liang, K. Ververis, K. W. Lim, A. Hung and T. C. Karagiannis, Front. Chem., 2020, 8, 1171.
18 P. Rao, R. Patel, A. Shukla, P. Parmar, R. M. Rawal, M. Saraf and D. Goswami, Mol. Diversity, 2021, 1-21.

19 Z. Shen, K. Ratia, L. Cooper, D. Kong, H. Lee, Y. Kwon, Y. Li, S. Alqarni, F. Huang and O. Dubrovskyi, bioRxiv, 2021, 1-13.

20 M. U. Mirza, S. Ahmad, I. Abdullah and M. Froeyen, Comput. Biol. Chem., 2020, 89, 107376-107383.

21 B. T. Freitas, I. A. Durie, J. Murray, J. E. Longo, H. C. Miller, D. Crich, R. J. Hogan, R. A. Tripp and S. D. Pegan, ACS Infect. Dis., 2020, 6, 2099-2109.

22 M. Kurosu, P. Narayanasamy, K. Biswas, R. Dhiman and D. C. Crick, J. Med. Chem., 2007, 50, 3973-3975.

23 N. Arunadevi, M. Swathika, B. P. Devi, P. Kanchana, S. S. Sundari, S. J. Kirubavathy, P. Subhapriya and E. R. Kumar, Surf. Interfaces, 2021, 24, 101094.

24 P. Dai, Russ. J. Coord. Chem., 2016, 42, 129-136.

25 J.-R. Liou, M. El-Shazly, Y.-C. Du, C.-N. Tseng, T.-L. Hwang, Y.-L. Chuang, Y.-M. Hsu, P.-W. Hsieh, C.-C. Wu and S.-L. Chen, Phytochemistry, 2013, 88, 67-73.

26 Y. Chen, L. Zhao and J. Jiang, Spectrochim. Acta, Part A, 2017, 175, 269-275.

27 Y. Takasaki and S. Takamizawa, Chem. Commun., 2015, 51, 5024-5027.

28 N. Arunadevi, P. Kanchana, V. Hemapriya, S. S. Sankaran, M. Mayilsamy, P. D. Balakrishnan, I.-M. Chung and P. Mayakrishnan, J. Dispersion Sci. Technol., 2021, 1-17.

29 P. Dai, E. Yang and X. Zhao, Russ. J. Coord. Chem., 2015, 41, 16-24.

30 S. H. Mousavi, M. R. Mohammadizadeh, Z. Roshan, A. Jamaleddini and S. Arimitsu, ACS Omega, 2020, 5, 18273-18288.

31 S. H. Mousavi, M. R. Mohammadizadeh, S. Arimitsu, D. Saberi, S. Poorsadeghi and K. Genta, RSC Adv., 2020, 10, 20552-20557.

32 S. A. Hashemi and M. R. Mohammadizadeh, ChemistrySelect, 2019, 4, 11995-12001.

33 A. Jamaleddini and M. R. Mohammadizadeh, Tetrahedron Lett., 2017, 58, 78-81.

34 M. R. Mohammadizadeh, D. Saberi and S. Z. Taghavi, Tetrahedron Lett., 2016, 57, 264-267.

35 S. A. Hashemi and M. R. Mohammadizadeh, ChemistrySelect, 2021, 6, 733-737.

36 N. Firoozi, Z. Roshan and M. R. Mohammadizadeh, Appl. Organomet. Chem., 2018, 32, e3963.

37 A. Jamaledini and M. R. Mohammadizadeh, Heteroat. Chem., 2018, 29, e21417.

38 T. Niu, S. Chen, M. Hong, T. Zhang, J. Chen, X. Dong and B. Ni, Green Chem., 2020, 22, 5042-5049.

39 E. Amadio, J. González-Fabra, D. Carraro, W. Denis, B. Gjoka, C. Zonta, K. Bartik, F. Cavani, S. Solmi and C. Bo, Adv. Synth. Catal., 2018, 360, 3286-3296.

40 L. Meng, W. Li, P. Guo, S. Wang and X. Tong, Catal. Commun., 2021, 154, 106305.

41 H. Luo, L. Wang, S. Shang, J. Niu and S. Gao, Commun. Chem., 2019, 2, 1-10.

42 E. L. Jackson, Org. React., 2004, 2, 341-375. 